\title{
Visualization of Merging Flow by Usage of PIV and CFD with Application to Grate-Kiln Induration Machines
}

\author{
I.A. S. Larsson ${ }^{1 \dagger}$, E. M. Lindmark ${ }^{1}$, T. S. Lundström ${ }^{1}$, D. Marjavaara ${ }^{2}$ and S. Töyrä ${ }^{2}$ \\ ${ }^{I}$ Division of Fluid Mechanics, Luleå University of Technology, SE-97187, Luleå, Sweden \\ ${ }^{2}$ LKAB, Kiruna, Sweden \\ †Corresponding Author Email: sofia.larsson@ltu.se
}

(Received December 2, 2010; accepted February 13, 2011)

\begin{abstract}
One way to upgrade iron ore is to process it into pellets. Such a process includes several stages involving complex fluid dynamics. In this work, focus is on the grate-kiln pelletizing process and especially on the rotary kiln, with the objective to get a deeper understanding of the aerodynamics in order to improve the combustion. A down-scaled, simplified model of the real kiln is created and both numerical and experimental analyses of the flow field are performed. Conclusions are that steady state simulations can be used to get an overview over the main features of the flow field. Precautions should though be taken when analyzing the recirculation zone since steady state simulations do not capture the transient, oscillating behavior of the flow seen in the physical experiment. These oscillations will under certain conditions considerably affect the size of the recirculation zone.
\end{abstract}

Keywords: Rotary kiln, Aerodynamics, CFD, PIV, Recirculation zone, Merging jets

\section{INTRODUCTION}

LKAB (Luossavaara-Kiirunavaara $\mathrm{AB}$ ) is an international minerals group that produces iron ore products for the steel industry. Their main product is iron ore pellets for production of hot metal in blast furnaces and direct reduction processes. The upgrade from crude ore to pellets takes place in an ore processing plant, i.e. sorting, concentrating and pelletizing plants. In the grate-kiln process, the pellets are first dried and pre-heated in the grate and then sintered in the kiln. The kiln is connected to an annular cooler which provides it with hot air, called secondary air, which is necessary for the combustion process. The mass flow of the secondary air is much higher than the primary air directly provided to the burner. Hence the secondary air is likely to have a large influence of the flow field within the kiln and of the performance of the burner. Therefore it is of interest to study the geometry of the tubings between the cooler and the kiln. A sketch of a grate-kiln induration machine is presented in Fig. 1.

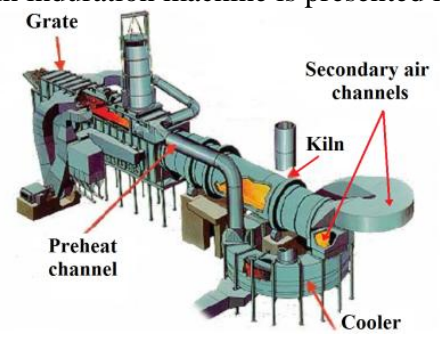

Fig. 1. Grate-kiln induration machine.
The induration process is multifaceted involving a highly turbulent, high temperature flow in intricate geometries. This makes it a challenge to study. Little is known about the flow field in the different parts of the process thus motivating deeper studies of the grate-kiln. Since fuel/air mixing largely controls the combustion process, the combustion air supply system and the resulting air flow patterns have a huge effect on the overall performance of the burner (Mullinger et al., 2008). The type of cooler, and its geometry, will define the secondary air flow regime and can result in severely distorted jet flows (and flames) (Mullinger et al., 2008), as will also be shown in this study. A stable flame is important for safe and efficient combustion, recognized by a constant point of ignition located very close to the burner nozzle. These conditions are crucially determined by the recirculation zone that forms between the secondary flows inlet jets (Mullinger et al., 2008). This zone is characterized by a rapid decay of the mean velocity compared to the surrounding flow field (Schneider et al., 2004) and it can be divided into a fuel driven recirculation zone created by the momentum from the fuel jet, and an air driven recirculation zone which, in this case, arises due to flow separation with the burner wall acting as a bluff body. Mullinger and Jenkins (2008) further explains the importance of this zone since it improves the flame stability as the reversed flow recirculates hot combustion products from downstream to mix with the incoming fuel stream and constantly ignites it. In this way the ignition point and flame are anchored to the burner nozzle which improves the combustion 
(Mullinger et al., 2008). If the recirculation zone is too large, the refractory lining can be damaged, and if the recirculation zone is too small it affects the mixing (Mullinger et al., 1996). Since mixing is the slowest part of the combustion process in a large, industrial flame, improving the mixing improves the combustion. The interaction between flames and vortices has been reviewed by (Renard et al., 2000) where they show how cylinders and structures in the flow can stabilize the flame.

Due to the design of the kiln hood, the flow field in the kiln will resemble the flow field seen when two parallel jets merge together. This flow field can be divided into three regions: the converging, merging and combined regions. The region closest to the dividing wall is called the converging region. As described by Lai et al. (1998), this region consists of a subatmospheric region as a result of the mutual entrainment of the jets, which causes the jets to curve towards each other. In the recirculation zone, the mean streamwise velocity is negative. The jets merge at the merging point, and downstream from this point the merging region is found. In this area the jets continue to interact and the mean streamwise velocity increases until it reaches its maximum value at the combined point. The distance to the merging point and combined point are known as merging length and combined length respectively. After the combined point, the combined region is found where the two jets combine and resemble one single jet.

In the present work the objective is to reveal flow features taking place in the kiln by using Computational Fluid Dynamics (CFD) on a simplified model of the real kiln, and validate different set-ups in the numerical model with physical experiments. There are at least two reasons to do this: to be able to decide if changes of the geometry up-stream of the burner can alter the flow field in a way that improves the combustion and reduces the NOx emissions, and to know how simple the set-up of the numerical simulation can be, still describing main features of the flow field. In the present study there will be no geometric changes since the aim is to start as simple as possible and lay grounds for future geometrical optimizations (Marjavaara et al., 2007). Later on more realistic geometries may be studied with the validated simulations as a base. The commercial code ANSYS CFX 11.0 is used for the numerical simulations and they are validated with experiments on a physical model using Particle Image Velocimetry (PIV). PIV is a non intrusive measuring technique that measures instantaneous velocity fields in arbitrary planes in any transparent fluid (Murzyn et al., 2006; Nordlund et al., 2007; Nordlund et al., 2010; Green et al., 2010). Using PIV to validate CFD simulations has previously been done by for example van Ertbruggen et al. (2008) and Ranade et al. (2001).

\section{MODEL DESCRIPTION}

A relatively simple down-scaled model of the kiln with the main features was built numerically for CFD simulations and in Plexiglas for validating experiments with PIV.

\subsection{Geometry}

The inlet tubes of the numerical model of the kiln are approximated as half circular pipes, see Fig. 2. To achieve a fully developed velocity profile before entering the kiln, the length of the inlet pipes are $4.0 \mathrm{~m}$ or 95 hydraulic diameters of the half circular pipe both in the virtual and physical model. Dean et al. (1976) obtained fully developed flow in a rectangular duct after 93.6 hydraulic diameters for $\operatorname{Re}=1 \cdot 10^{5}$ (based on the height of the duct). To the authors knowledge similar results have not been presented for flow through half circular pipes.

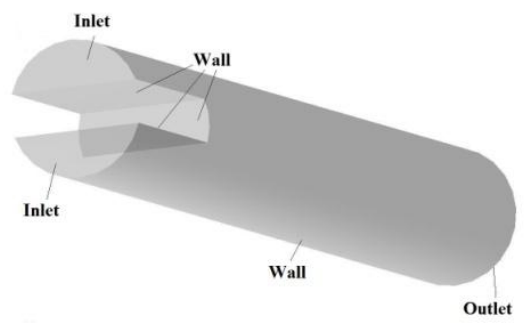

Fig. 2. Model geometry with boundaries (the full length of the inlet pipes is not shown).

The kiln is modeled as a straight, non-rotating, horizontal cylinder without pellets, while the real kiln has a smaller expansion in diameter, an inclination angle to the ground and rotates. The burner is excluded since only the cold flow field without combustion is of interest in the present investigation.

\subsection{CFD Set-up}

The geometry is imported into ANSYS ICEM and a mesh built from hexahedral elements is created. An ogrid consisting of approximately 12.5 million nodes is designed in order to get a good mesh adaptation around the round edges. The $\mathrm{y}^{+}$values are in the range of 0.001 to 6 , with an area averaged value of approximately 1.3 , so the boundary layer is not fully resolved everywhere in the geometry according to the requirements of lowRe wall formulations for an $\omega$-based turbulence model (Ansys CFX-Solver Theory Guide, 2006). Since the main interest in this investigation is the bulk flow, the areas with high $\mathrm{y}^{+}$values do not affect the results. A mesh study was performed with $\mathrm{Re}=1 \cdot 10^{4}$ and with the efficiency factor as the dependent variable, defined as the ratio of mass averaged total pressure at the inlet to that at the outlet (Casey et al., 2000). A conclusion from this study is that a mesh consisting of at least 3 million nodes is needed in order to achieve a grid independent solution. The reason for using 12.5 million nodes in the simulations is to resolve the boundary layer for higher Reynolds number. In order to maintain the mesh quality, more nodes are placed in the flow direction to reduce the distance between the nodes in that direction, and to keep the aspect ratio within an acceptable range.

Three dimensional steady state, Reynolds averaged Navier-Stokes equations closed by the Reynolds stress turbulence model BSL are solved for the turbulent flow field. One reason for using a second moment closure model is that it reveals secondary flows (flow in the 
transverse plane, perpendicular to the mean axial flow) which arise in the non-circular inlet pipes. A standard two-equation model does not show this flow feature (Speziale, 1982). Interestingly, comparisons with initial simulations using two-equation models yielded no noticeable increase of simulation time or convergence problems with the more advanced turbulence model.

A plug profile is set at each inlet and at the outlet an average static pressure is employed with a relative pressure of zero $\mathrm{Pa}$, averaged over the whole outlet. A second order discretization scheme is used for the advection term. The convergence criterion is RMS residuals below $10^{-6}$ (Ansys CFX-Solver Theory Guide, 2006) and therefore double precision is used. Isothermal conditions are assumed so the energy equations are not applied. The simulations are partly carried out on a 150-node PC-cluster. It has been demonstrated that the CFD-code applied parallelize excellent on this cluster (Hellström et al., 2007).

\subsection{Experimental Set-up}

The experimental kiln has the same geometry as the virtual model, described in the previous section. To control the flow rate in the half circular pipes the flow is monitored with magnetic flow meters (Krohne Optiflux DN50), and the temperature in the set-up is monitored with a Pt100. The temperature of the water in the set-up was controlled to $20 \pm 0.4{ }^{\circ} \mathrm{C}$ with a cooling system in the tank. The turbulence intensity in the inlet pipes was approximately $9 \%$.

The PIV-system used is a commercially available system from LaVision $\mathrm{GmbH}$. It consists of a Litron Nano L PIV laser, i.e. a double pulsed Nd: YAG with a maximum repetition rate of $100 \mathrm{~Hz}$, and a LaVision FlowMaster Imager Pro CCD-camera with a spatial resolution of $1280 \times 1024$ pixels per frame. The laser is mounted on a traverse so that the laser sheet and camera can be repositioned up to $500 \mathrm{~mm}$ in the $\mathrm{x}-$, $\mathrm{y}-$ and $\mathrm{z}-$ directions. The tracer particles used are hollow glass spheres with a diameter of $6 \mu \mathrm{m}$ from LaVision $\mathrm{GmbH}$.

PIV measurements are performed with a frequency of $80 \mathrm{~Hz}$ during 6 seconds which implies that for each measurement 480 picture pairs are produced. In order to be able to compare the measurements to results from steady state simulations, the 480 frames are timeaveraged. Time between laser pulses was $400 \mu$ s, which resulted in a particle displacement of 8 pixels in the streamwise velocity direction. Raffel et al. (2007) recommend that particles should move $1 / 4$ of the interrogation window; larger displacements result in too large loss in particle matching. All in all this boils down to an appropriate interrogation window of $32 \times 32$ pixels.

Measurements are taken in four positions in the flow direction from the half circular pipes and downstream within the kiln. At each position measurements are performed on three planes directed along the flow, one in the middle and one on each side $25 \mathrm{~mm}$ from the middle. The order in which the positions are measured is randomized. In the processing of the PIV measurements first a Gaussian filter is applied to improve the accuracy of the particle location. A multipass scheme with decreasing window size and window offset is then used to calculate the displacement of the particles. The interrogation window size was $64 \times 64$ pixels decreasing to $32 \times 32$ pixels both with an overlap of $50 \%$.

The overall measurement accuracy in PIV is a combination of a variety of aspects extending from the recording process all the way to the methods of evaluation (Raffel et al., 2007). A cornerstone in all experimental design is to randomize the experimental procedure. By proper randomization, the effects of the uncertainties are averaged out. The measurement uncertainty consists of uncertainty due to biased errors and precision errors (or measurement errors) (Lindmark, 2008). The seeding particles should be small enough to follow the fluid flow well and they should also scatter the laser light effectively. In order to determine the velocity the particle displacement between two images must be computed. Together with the time between the exposures, a two-dimensional velocity field can be calculated. The particle displacement should not be too large, as outlined earlier in this section, so the time between exposures and the thickness of the laser sheet needs to be optimized in order to maximize the displacement (within the recommended range) and minimize the particle loss out of the laser sheet. Sources of errors that affect the measurement accuracy include refraction on surfaces, camera repositioning accuracy and characteristics of the optical components. Finally, the evaluation with the cross-correlation and other computed functions in the post-processing may give rise to further sources of error (Kirschner et al., 2007).

\subsection{Numerical and Experimental Settings}

The flow in the real kiln has a Reynolds number of approximately $8 \cdot 10^{5}$ based on the diameter of the kiln. In the experiment a Reynolds number of $5 \cdot 10^{4}$ and $1 \cdot 10^{5}$ is used, which corresponds to a mass flow of 3.95 $\mathrm{kg} / \mathrm{s}$ and $7.9 \mathrm{~kg} / \mathrm{s}$ respectively in the model kiln. The magnetic flow meters control the flow to an accuracy of $0.1 \%$ in the experiments. The fluid chosen in the physical model is water, allowing the smaller scale of the model kiln. Three distributions between the inlet pipes are evaluated, namely 50/50, 40/60 and 70/30 in order to detect how the distribution of mass flow affects the flow field in general and the recirculation zone in particular.

The laser cannot illuminate the entire measuring section at once so it has to be moved with the traverse to cover the area of interest. Hence several frames are linked together after each other to create a complete picture. This result in non-continuous plots in the figures that are due to: difficulties with overlapping the different frames into one picture, insufficient laser illumination at the borders of the measuring areas and joints in the geometry, especially where the inlet pipes are connected to the kiln. Getting perfect optical availability at such a joint is rather tricky. These flaws do not, however, affect the results presented in this paper. 


\section{RESUlTS AND DISCUSSION}

To start with, a comparison of the streamwise velocity profile in two planes with equal off-set from the main axis of the kiln yields that the flow field is symmetric about a vertical plane in the middle of the kiln and directed along the flow. This is exemplified with velocity profiles (normalized with the bulk velocity and the kiln diameter) at the merging point in Fig. 3. As can be seen, there is no noticeable difference between the two planes, thus indicating that the flow field is symmetric.

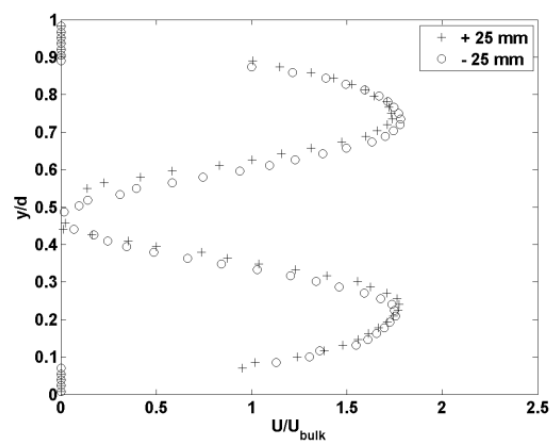

Fig. 3. Velocity profile at the merging point in the kiln taken at an offset of $25 \mathrm{~mm}$ from the center plane.

Equal flow distribution between the inlet pipes. Reynolds number $10^{5}$ in the kiln.

In order to derive the importance of applying the correct full-scale Reynolds number in the model, a comparison of the streamwise velocity profile in the merging point for $\operatorname{Re}=5 \cdot 10^{4}$ and $1 \cdot 10^{5}$ is performed for the $50 / 50$ distribution between the two inlet pipes. The result shows that the profiles do not differ between the two Reynolds numbers (Fig. 4). This indicates that an exact agreement of Reynolds numbers is not important as long as it is within the fully turbulent range, agreeing with the results reported by Yin et al. (2007). Hence, when measuring the 70/30 distribution the Reynolds number is set to $5 \cdot 10^{4}$ for the practical reason that the pump used in the experiments cannot manage that distribution at $\operatorname{Re}=1 \cdot 10^{5}$.

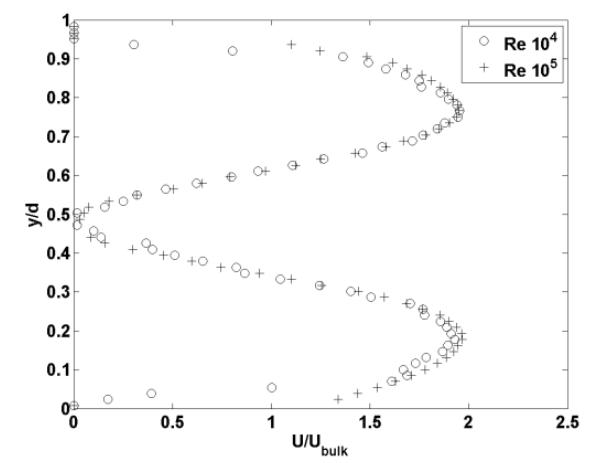

Fig. 4. Velocity profiles at the merging point for two different Reynolds numbers with equal distribution between the inlet pipes.

The effect of gravity on the flow field is also investigated with usage of the PIV measurements by first pumping $60 \%$ of the mass flow through the top inlet pipe and then through the lower one. The streamwise velocity profile in the merging point for the two cases are practically mirror images of each other, see Fig. 5. This indicates that gravity does not need to be taken into consideration when performing the simulations.

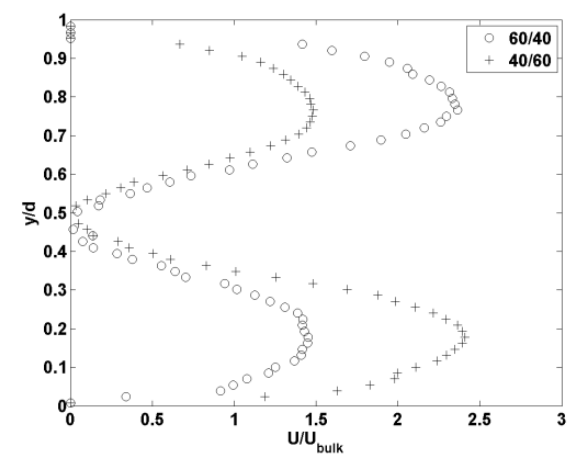

Fig. 5. Velocity profiles at the merging point for different distributions in top and bottom inlet pipe. Reynolds number $10^{5}$ in the kiln.

Simulation results based on a stationary set-up can be seen in Figs. 6-8 where velocity vectors are shown for the distributions 50/50, 40/60 and 70/30. Timeaveraged experimental results can also be seen in the same figures for a qualitative comparison. To compare simulations with experimental results quantitatively, the centerline velocity is plotted (Fig. 9). Where the centerline velocity reaches its maximum value the combined point can be found and as can be seen in the graph, the predicted centerline velocity in the simulations peaks later (outside of the measuring area) than the experimental results. This implies that the inlet jets travel further into the kiln and that the combined point is located farther downstream in the simulations than in the experiments.

This may be explained by the transient behavior of the flow field in the experiments. The flow is oscillating in reality which improves the mixing of the two inlet jets and lessens their strength. This behavior is not captured with steady state simulations, allowing the jets to more undisturbed reach farther downstream the kiln. The largest amplitudes of the oscillations in the experiments are found when the mass flow distribution between the inlet pipes is equal. When one of the inlet jets starts to become dominant the other jet is pulled towards the dominant one and the flow field is stabilized, agreeing with the results reported by Bunderson et al. (2005). This can clearly be seen in the experimental case with the 70/30 distribution where the conformity between simulations and physical experiments is rather good, see Fig. 9.

The frequency of the large-scale oscillations can easily be found from Fourier transforms of the velocity magnitude. Figs. 10-12 shows FFT power spectra of the velocity in the $y$-direction for the three different cases, taken in a point in the recirculation zone $(x=143 \mathrm{~mm}$, $\mathrm{y}=57 \mathrm{~mm}$ ). One prominent peak around $12 \mathrm{~Hz}$ can be seen in the 50/50 and 40/60 cases while the 70/30 case shows no dominating frequency. The $12 \mathrm{~Hz}$ peak corresponds to the large scale oscillations that make the flow field highly unsteady, making comparisons with steady state simulations hard. Notice that the amplitude of the peak in the 50/50 case is almost 5 times the 
amplitude in the 40/60 case, indicating larger oscillations of the velocity field.
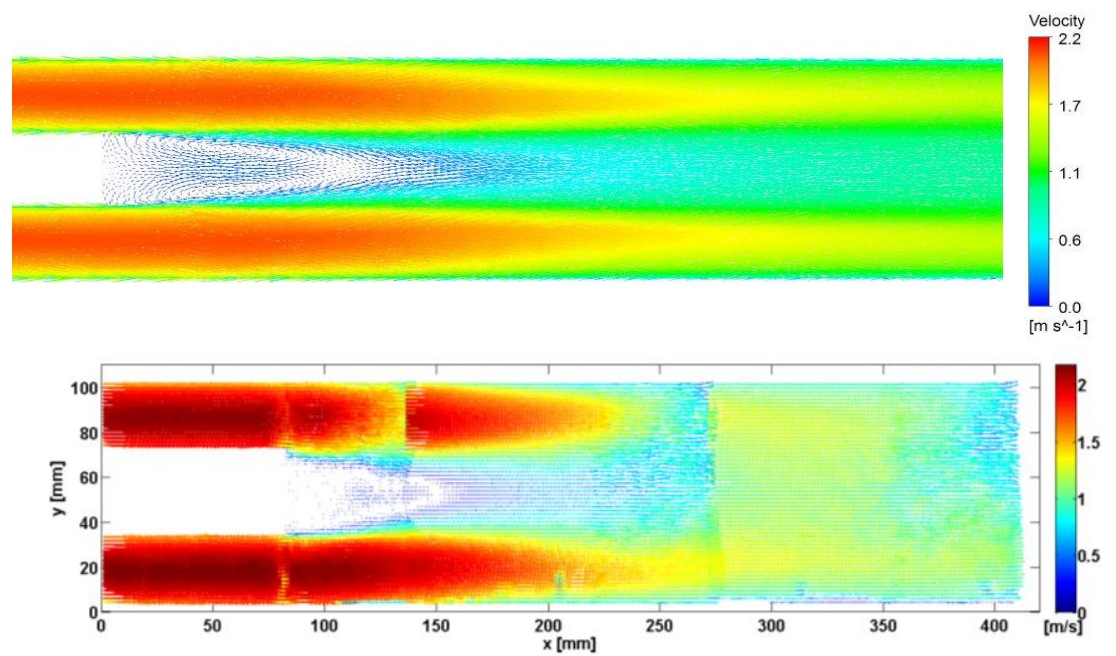

Fig. 6. Equal distribution in the inlet channels, steady state simulation results atop, time-averaged experimental results down below. Reynolds number $10^{5}$ in the kiln.
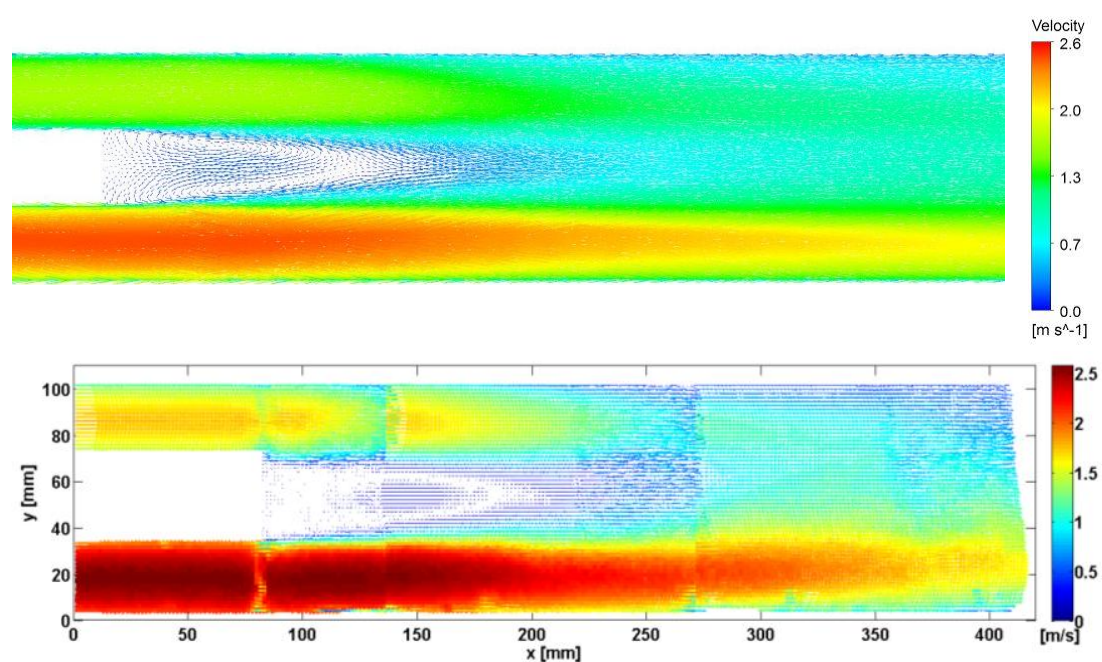

Fig. 7. 40/60 distribution in the channels, steady state simulation results atop, time-averaged experimental results down below. Reynolds number $10^{5}$ in the kiln.
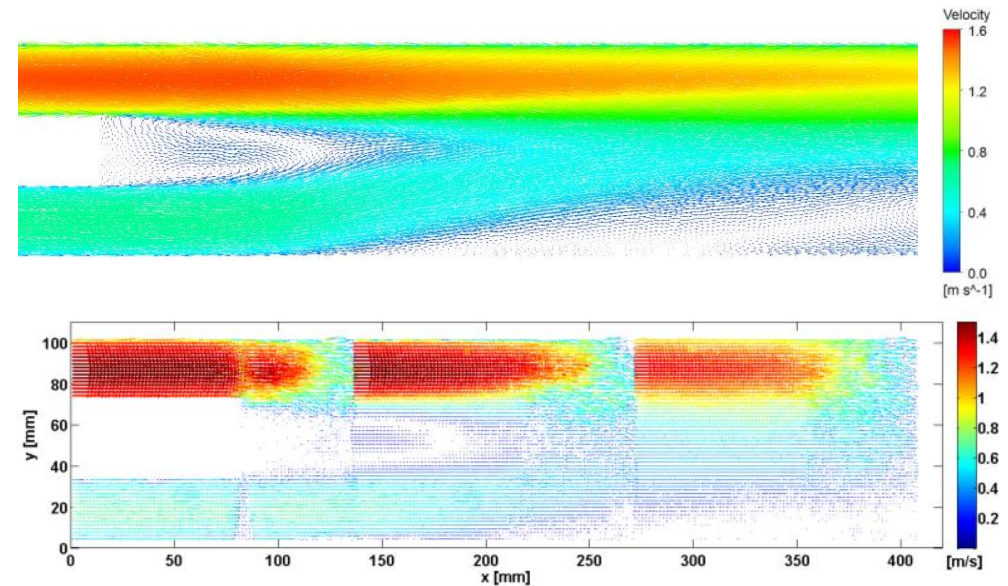

Fig. 8. 70/30 distribution in the channels, steady state simulation results atop, time-averaged experimental results down below. Reynolds number $5 \cdot 10^{4}$ in the kiln. 


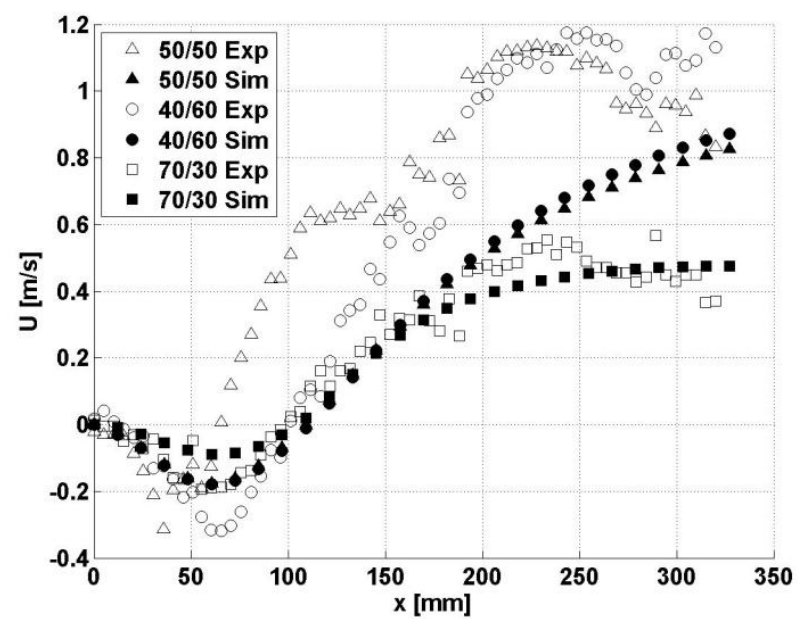

Fig. 9. Velocity along the centreline of the kiln for three different distributions in the inlet pipes: 50/50, 40/60 and 70/30. Results from both experiments and simulations.

Table 1 Length of the recirculation zone at different mass flow distributions between the inlet pipes

\begin{tabular}{|c|c|c|c|}
\hline $\begin{array}{l}\text { Length } \\
\text { RZ }(\mathbf{m m})\end{array}$ & $\mathbf{5 0 / 5 0}$ & $\mathbf{4 0 / 6 0}$ & $\mathbf{7 0 / 3 0}$ \\
\hline Experiment & 64 & 100 & 100 \\
\hline Simulation & 109 & 111 & 104 \\
\hline
\end{tabular}

One measurement of particular interest is the length of the recirculation zone (RZ) that takes shape in between the two inlet jets, in front of the wall where the burner is located in the real kiln. The recirculation zone is important for the flame stability as outlined in the introduction. The length of this zone can be derived from the centerline velocity. The merging point where the two jets merge together can be found where the centerline velocity transit from negative to positive values. This point is also the end of the recirculation zone, and hence a length can be derived. Agreement between simulations and experiments are best when one of the inlet jets is dominant, case 70/30, see Table 1 . In the $50 / 50$ case the length of the recirculation zone is about $70 \%$ longer in the simulations than in the experiments. This is because of the large amplitudes of the oscillations, a feature which is missed in steady state simulations. The length of the recirculation zone is thus a clear measurement of the effect of the oscillating behavior and it is obvious that the oscillations improve the mixing of the two jets and reduce the recirculation zone. The flat region that can be seen between $x=100$ $\mathrm{mm}$ and $\mathrm{x}=150 \mathrm{~mm}$ is due to the previously described difficulties with illuminating the entire measuring area at once. Several frames are linked together into one figure which can clearly be seen in the experimental results in Figs. 6-8. Decreased laser illumination at the border between two frames creates this deviation.

To further visualize the behavior of the merging jets in the kiln, streamlines are presented at different phases. Phase average presentation of streamlines behind a cylinder has been presented by Perrin et al. (2006), where the cylinder is confined in a narrow surrounding with high blockage coefficient $(\mathrm{D} / \mathrm{H}=0.208)$. The vortex structure behind the cylinder has a Strouhal number of 0.21 , the same result is presented by Djeridi et al. (2003) in a similar arrangement. In the present set-up the frequency of the vortex shedding was 11.88 $\mathrm{Hz}$ (see Fig. 10) corresponding to a Strouhal number of 0.23 based on the frequency of the alternating vortices in the kiln with even distribution in the inlets, the length between the inlets and the mean velocity in the inlet pipes. The phase average result, together with vorticity contour plots are presented in Fig. 13 at phases $\varphi=0^{\circ}$, $\varphi=45^{\circ}, \varphi=90^{\circ}, \varphi=135^{\circ}, \varphi=180^{\circ}, \varphi=225^{\circ}, \varphi=$ $270^{\circ}$ and $\varphi=315^{\circ}$. Each phase is a mean over approximately $14-15$ pictures belonging to that phase +/- 5 degrees. The results clearly show the alternating vortices travelling downstream into the kiln. The similarity with flow past a bluff body, for example a cylinder, is obvious.

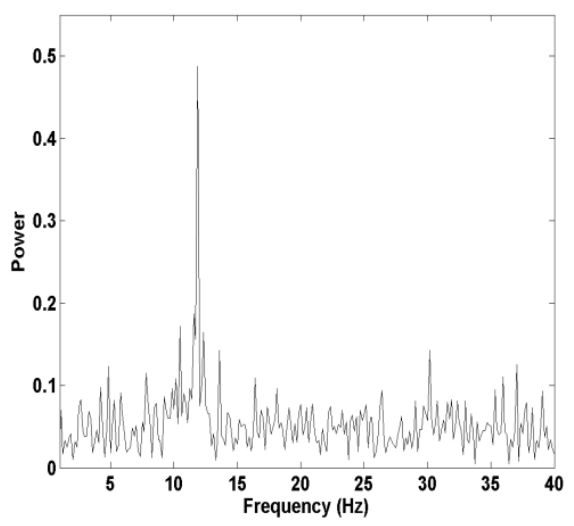

Fig. 10. Fourier transform of 50/50 distribution $y$ direction velocity in $(x, y)=(143,57) \mathrm{mm}$. 


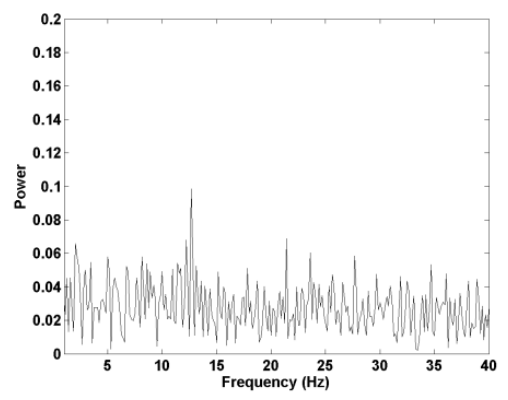

Fig. 11. Fourier transform of $40 / 60$ distribution $y-$ direction velocity in $(x, y)=(143,57) \mathrm{mm}$.

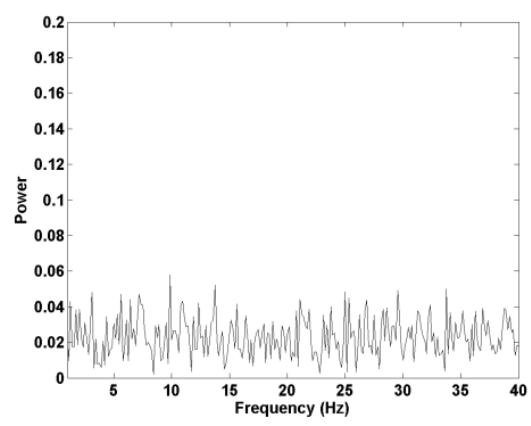

Fig. 12. Fourier transform of 70/30 distribution ydirection velocity in $(\mathrm{x}, \mathrm{y})=(143,57)$.

One thing that differs when comparing the flow field in the kiln with flow past a cylinder is the length of the recirculation zone. When a cylinder is present the flow is deflected more compared to the flow in the kiln where a vertical wall divides the two jets (Sheridan et al., 1997). The increased deflection results in a shorter recirculation zone, as presented by Erdem et al. (2002).
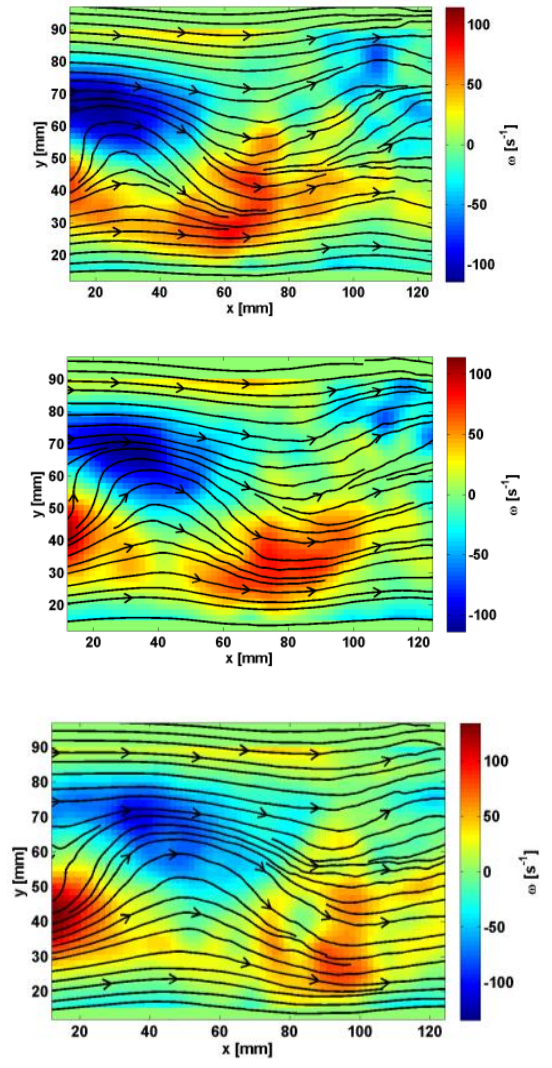
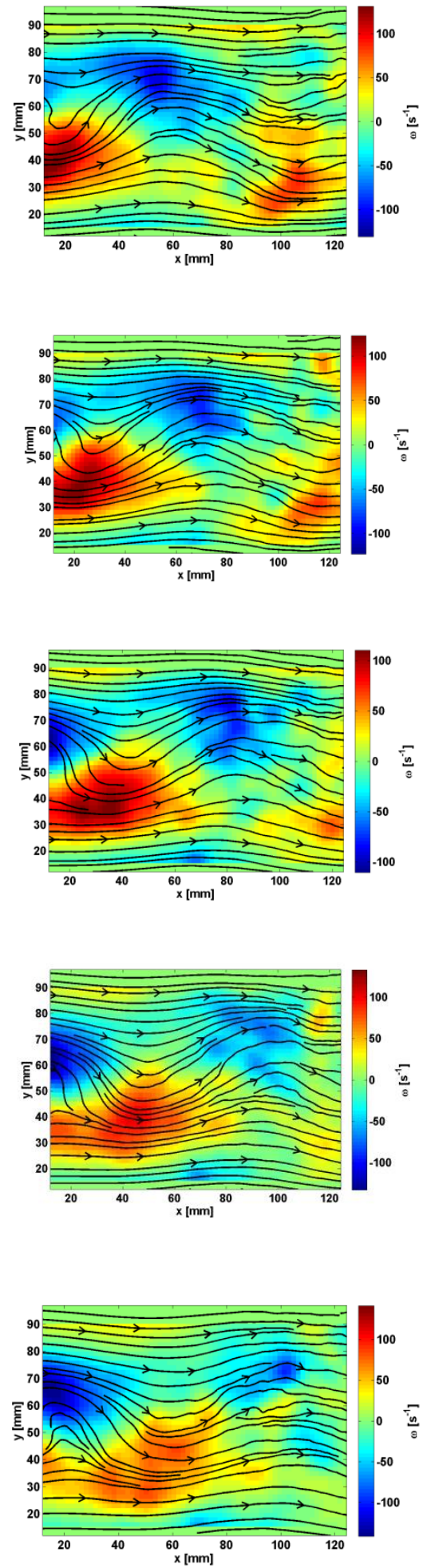

Fig. 13. Phase averaged streamlines and vorticity contours for flow with equal distribution between the inlet pipes at Reynolds number $10^{5}$. Phases $\varphi=0^{\circ}, \varphi=$ $45^{\circ}, \varphi=90^{\circ}, \varphi=135^{\circ}, \varphi=180^{\circ}, \varphi=225^{\circ}, \varphi=270^{\circ}$ and $\varphi=315^{\circ}$, from top to bottom. 


\section{Conclusions}

Physical experiments and simulations show that the flow characteristics are strongly dependent on the flow distribution between the secondary flow channels (50/50, 40/60, 70/30). With equal distribution oscillations with large amplitudes are seen in the kiln in the experiments. The amplitude decreases when one of the inlet jets becomes dominant and the smallest oscillations can be seen in the 70/30 case. The dominant jet attracts the other one and stabilizes the flow field. Since the shape of the recirculation zone including the location of the merging point is directly dependent on these oscillations it is likely that also the combustion is affected. The oscillations influence, furthermore, the flow downstream the merging point by increasing the mixing of the two jets and reducing the combined length.

In general, the steady state simulations show good qualitative agreement with the time-averaged results from the physical experiments. The main features of the flow field are captured and the simulation time is short compared to a transient simulation on the same mesh. Best agreement is obtained for the 70/30 case where also the quantitative agreement is good. The lack in conformity for the 50/50 case can be traced to the fact that oscillations are not captured in the steady state simulations. Hence for cases with strong oscillations transient simulations should be carried out. The flow field and the recirculation zone are not affected if Reynolds number is lowered from $1 \cdot 10^{5}$ to $5 \cdot 10^{4}$. Hence there is no need for exact agreement of the Reynolds number between model and reality as long as the flow is fully turbulent. Gravity does not affect the flow field pattern or the recirculation zone; therefore gravity can be neglected in the simulations. For the case studied it is shown that a more advanced turbulence model can be applied. Not necessarily because it is required but because computational costs does not increase in a noticeable way.

Future work includes further analysis and comparison between simulations and experiments. Much information was retrieved in the experiments which can be used for further validation. In an ongoing work transient simulations are carried out in order to compare time-resolved simulations and experiments. Performing further PIV and LDV measurements in other planes will also be done in the future. This in order to experimentally visualize the secondary flow (flow in the transverse plane, perpendicular to the mean axial flow) arising in the half circular pipes. Changing the inlet pipes angles to see how the incoming flow angle affects the flow field is another future project.

\section{ACKNOWLEDGEMENTS}

This work was carried out within the framework of the Faste Laboratory, a VINNOVA Excellence center. The authors also acknowledge discussions with Prof. Graham Nathan from the University of Adelaide and with LKAB. LKAB also partly financed the work via the Faste Laboratory.

\section{REFERENCES}

Ansys CFX-Solver Theory Guide (2006), Elsevier Academic Press.

Bunderson, N.E. and B.L. Smith (2005). Passive mixing control of plane parallel jets. Experiments in Fluids, 39, 66-74.

Casey, M. and T. Wintergerste (2000). Best Practice Guidelines. Special Interest Group on "Quality and Trust in Industrial CFD”, 1 ed.

Dean, R.B. and P. Bradshaw (1976). Measurements of interacting turbulent shear layers in a duct. $J$. of Fluid Mechanics, 78(4), 641-676.

Djeridi, H., M. Braza, R. Perrin, G. Harran, E. Cid and S. Cazin (2003). Near-Wake Turbulence Properties around a Circular Cylinder at High Reynolds Number. Flow, Turbulence and Combustion, 71, $19-34$.

Erdem, G. and V. Ath (2002). Interaction of two parallel rectangular jets. Proceedings from the 23rd Congress of International Council of the Aeronautical Sciences, 8-13 September, 2002, Toronto, Canada. Paper ICAS 2002-R15.

Green, T., E.M. Lindmark, T.S. Lundström and L.H. Gustavsson (2010). Flow characterization of an attraction channel as entrance to fishways. River Research and Application, Published on-line DOI: 10.1002/rra.1426

Hellström, G. B.D. Marjavaara and T.S. Lundström (2007). Redesign of a Hydraulic Turbine Draft Tube with aid of High Performance Computing. Advances in Engineering Software, 38 (5), 338344

Kirscher, O. and A. Ruprecht (2007). Velocity measurements with PIV in a straight cone draft tube. Proceedings of the $3^{\text {rd }}$ German-Romanian Workshop on Turbo machinery Hydrodynamics, 10-12 May 2007 Timisoara, Romania.

Lai, J.C.S. and A. Nasr (1998). Two parallel plane jets: comparison of the performance of three turbulence models. Proceedings of the Institution of Mechanical Engineers, 212 (6), 379-391.

Lindmark, E.M. (2008). Flow design for migrating fish. Doctoral Thesis, Luleå University of Technology, Sweden. ISSN: 1402-1544.

Mullinger, P.J. and B.G. Jenkins (1996). Optimizing kiln efficiency and emissions using combustion process modeling. In: Dhir, K.R. and Dyer, T.D. (Ed.), Proceedings of the international conference Concrete in the Science of Mankind, 27-28 June 1996 Dundee, Scotland. London, UK: E \& FN Spon, 377-387. 
Mullinger, P. and B. Jenkins (2008). Industrial and process furnaces: principles, design and operation. 1st ed. Oxford, UK: ButterworthHeinemann.

Murzyn, F., D. Mouaze and J.R. Chaplin (2006). Flow visualization and free surface length scales measurements in a horizontal jet beneath a free surface. Experimental Thermal and Fluid Science, 30, 703-710.

Marjavaara, B.D., T.S. Lundström, T. Goel, Y. Mack and W. Shyy (2007). Hydraulic diffuser shape optimisation by multiple surrogate model approximations of Pareto fronts. Journal of Fluids Engineering, 129 (9), 1228-1240.

Nordlund, M., S.P. Fernberg and T.S. Lundström (2007). Particle deposition mechanisms during processing of advanced composite materials. Composites Part A: Applied Science and Manufacturing, 38(10), 2182-2193.

Nordlund, M. and T.S. Lundström (2010). An investigation of particle deposition mechanisms during impregnation of dual-scale fabrics with Micro Particle Image Velocimetry. Polymer Composites. DOI: 10.1002/pc.20910.

Perrin, R., M. Braza, E. Cid, S. Cazin, F. Moradei, A. Barthet, A. Sevrain and Y. Hoarau (2006). NearWake Turbulence Properties in the High Reynolds Number Incompressible Flow Around a Circular Cylinder Measured by Two- and ThreeComponent PIV. Flow, Turbulence and Combustion, 77, 185-204.

Raffel, M., C.E. Willert, S.T. Wereley and J. Kompenhans (2007). Particle Image VelocimetryA practical guide. Berlin, Germany: SpringerVerlag.

Ranade, V.V., M. Perrard,N. Le Sauze, C. Xuereb and J. Bertrand (2001). Trailing vortices of rushtone turbine: PIV Measurements and CFD Simulations with Snapshot Approach. Trans IChemE, 79, Part A, January 2001.

Renard, P.H., D. Thévenin, J.C. Rolon and S. Candel (2000). Dynamics of flame/vortex interactions. Progress in Energy and Combustion Science, 26, 225-282.

Schneider, G.M., G.J. Nathan and T. O'Doherty (2004). Implications on flame stabilization in a precessing jet flame from near field velocity measurements. Proceedings of the Institution of Mechanical Engineers, 218, 677-687.

Sheridan, J., J.C. Lin and D. Rockwell (1997). Flow past a cylinder close to a free surface. Journal of Fluid Mechanics, 330, 1-30.
Speziale, C.G. (1982). On turbulent secondary flows in pipes of noncircular cross-section. Int. J. Engng. Sci., 20(7), 863-872.

Van Ertbruggen, C., P. Corieri, R. Theunissen, M.L. Riethmuller and C. Darquenne (2008). Validation of CFD predictions of flow in a 3D alveolated bend with experimental data. Journal of Biomechanics, 41, 399-405.

Yin, Z.Q., H.J. Zhang and J.Z. Lin (2007). Experimental study on the flow field characteristics in the mixing region of twin jets. Journal of Hydrodynamics, Ser. B, 19(3), 309-313 